\title{
On mixed convection in a cavity with sinusoidally heated moving lid and uniformly heated and cooled side walls
}

\section{Deborah S Ducasse and Precious Sibanda*}

\section{"Correspondence:}

sibandap@ukzn.ac.za

School of Mathematics, Statistics and Computer Science, University of KwaZulu-Natal, Private Bag X01 Scottsville, Pietermaritzburg, 3209, South Africa

\section{Springer}

\begin{abstract}
In this study a steady state two-dimensional mixed convection problem in an air filled cavity is investigated. The effects of linearly heated and uniformly cooled walls on flow and heat transfer characteristics within the cavity are determined. The strength of the fluid circulation within the cavity is found for both heated and cooled walls for different Reynolds and Grashof numbers. The nonlinear coupled equations are solved numerically using the penalty-Galerkin finite element method. Stream function and isotherm results are obtained for different Reynolds and Grashof numbers. The results for the heat transfer rate are presented in terms of both the local and the average Nusselt number. In general, the strength of the circulation is stronger for the case of cooled walls, and the anti-clockwise circulation is significantly stronger for cooled walls, while the clockwise circulation is only slightly stronger for the cooled walls and the difference in strength decreases both with increasing Reynolds and Grashof numbers. Both the local and average Nusselt numbers are generally higher for the case of cooled side walls than that for heated side walls.

MSC: 34B15;65N30; 76M20
\end{abstract}

\section{Introduction}

Over the last decade mixed convection in a square cavity has become an increasingly attractive field of study. The popularity of such studies is due in part to the many applications of such flows in industrial and natural settings. These applications include, for example, the thermal-hydraulics of nuclear reactors, drying technologies, the dynamics of lakes, and food processing. Mixed convection in a lid-driven cavity has been extensively studied. Torrance et al. [1] investigated the fluid motion in a lid-driven cavity where the upper wall was maintained at a different temperature to that of the other walls in the cavity. They observed that an increase in the aspect ratio led to an increase in secondary circulations in the lower part of the cavity.

Kawaguti [2] was one of the first to investigate the effect of the Reynolds number on forced convection in a square cavity. Results were found using finite difference methods for values of the Reynolds number $R e$ between 0 and 64 for different ratios of lengths of the cavity. An attempt was made to find results for $R e=128$, but a convergent solution was not found. By modifying Kawaguti's method, Burggraf [3] was able to attain stable solutions for forced convection for a Reynolds number up to 1,000. Heinrich and Marshall [4] used the penalty finite element method to investigate lid-driven cavity flow up to $R e=400$. For a

(C) 2013 Ducasse and Sibanda; licensee Springer. This is an Open Access article distributed under the terms of the Creative Commons Attribution License (http://creativecommons.org/licenses/by/2.0), which permits unrestricted use, distribution, and reproduction in any medium, provided the original work is properly cited. 
low Reynolds number, their results were in excellent agreement with published results. As the Reynolds number increased, however, their results were less accurate in comparison to other results. Early studies of natural convection in a cavity were of an experimental nature. An example of an early experimental study is that of Eckert and Carlson [5]. They made an experimental investigation into the flow and heat transfer in an enclosed cavity. The top and bottom plates were heated to different temperatures. Their results showed that, contrary to previous beliefs, it was very unlikely that a core of uniform temperature could exist at large Rayleigh numbers unless the height to width aspect ratio was very small. Ostrach [6] gives an excellent review of early experimental studies of convection in a cavity. Recently, Prasad and Koseff [7] investigated mixed convection in a lid-driven cavity through experimental means where the lower surface was heated and the upper surface cooled. The lid speed and values of the Reynolds and Grashof numbers were varied. Their results indicated that the overall heat transfer rate was a very weak function of the Grashof number for the range of Reynolds numbers examined.

Moallemi and Jang [8] investigated flow in a lid-driven cavity with the bottom wall heated. They studied the effects of small Prandtl numbers on the flow and heat transfer for various values of the Richardson number. Sivakumar et al. [9] investigated mixed convection in a lid-driven cavity with a cooled right wall and a heat source in the left wall. Three different lengths of the heat source were examined. The finite volume method was used to solve the resulting equations. It was found that for low values of the Richardson number, reducing the heating portion length had no effect on the fluid flow. However, on increasing the Richardson number, it was seen that the flow depended heavily on the length of the heat source. It was also found that reducing the length of the heated portion of the wall resulted in a better heat transfer rate. Nithyadevi et al. [10] studied the case where the side walls were partially heated or partially cooled while the rest of the cavity was well insulated. They used the finite volume method to solve the equations. They studied nine different cases where the position of the heating and cooling sources was moved between the top, bottom and middle of the wall of the cavity. They found that the heat transfer rate was enhanced when there was a cooling source near the top of the cavity. For a combination of a bottom top heating source, the heat transfer rate was found to be high, while for the combination of a top bottom heating source, the heat transfer rate was low. Related studies have been done by, among others, Paraconi and Corvaro [11] and Pesso and Piva [12], Sathiyamoorthy et al. [13].

Recently, Basak et al. $[14,15]$ studied mixed convection in a lid-driven cavity. The moving upper lid was insulated in both studies. In Basak et al. [14] they investigated cooled side walls with a heated (uniformly and non-uniformly) bottom wall. In Basak et al. [15] they looked at a uniformly heated bottom wall with linearly heated left side wall. The right side wall was heated either linearly or cooled uniformly. Both papers used the penalty finite element method to solve the governing equations of motion and heat transfer. Both studies found that the strength of convection increased with increasing Grashof numbers and that lid-driven flow was dominant for $G r=10^{3}$. Their work showed that the heat transfer rate for a uniformly heated bottom wall was higher than that for the non-uniformly heated bottom wall and that the heat transfer rate was larger for the case of one cooled right wall than it was for the case when both walls were linearly heated.

Research has also been done into mixed convection with more than one moving wall in a cavity. Oztop and Dagtekin [16] used the finite volume method to investigate three 
cases of moving walls with adiabatic top and bottom wall and cooled left wall and heated right wall. They observed that the fluid flow and the heat transfer in the cavity depended on both the Richardson number $R i$ and the direction of motion of the walls.

Cheng and Liu [17] investigated four cases of mixed convection in a square cavity. In the first case, the side walls were well insulated while the top moving wall was heated and the bottom wall cooled. In the second case, the bottom wall was heated and the top wall cooled. In the third and fourth cases, the top and bottom walls were well insulated and one side wall was cooled while the other was heated. They found that for the first case, when the Richardson number was greater than 1 , the heat transfer was mainly through conduction. When the Richardson number was less than 1, forced convection dominated the fluid flow as was found by Oztop and Dagtekin [16]. In contrast to the findings of Oztop and Dagtekin, in the second case, when the Richardson number was greater than 1 , the lower half of the cavity showed natural convection while the upper part was dominated by forced convection. When the Richardson number was less than 1, the fluid flow was similar to that for case 1 . For the third case when the Richardson number was greater than 1 , heat transfer was shown to be by conduction near the side walls and by convection in the center. Again when the Richardson number was less than one, the fluid flow was similar to that for cases 1 and 2. For the fourth case, a large portion of the cavity was dominated by natural convection for all values of the Richardson number.

Corcione [18] investigated the effect of the Rayleigh number and the width to height aspect ratio of the cavity on steady laminar natural convection in an air filled cavity. The cavity was heated from below and cooled from above while six different cases of side wall heating and cooling were investigated. They showed that the heat transfer rate from a cooled or heated boundary increased as the Rayleigh number increased. In the case with insulated side walls, the heat transfer rate from the heated bottom wall or top cooled wall increased as each of the side walls was replaced by a cooled or heated side wall. Wong [19] investigated mixed convection in a square cavity. The left wall was maintained at a constant velocity. The other walls were kept stationary. The top and bottom walls were insulated and the left moving wall was heated while the right side wall was cooled. A numerical solution was found using the consistent splitting scheme and the finite element method. Wong fixed the Reynolds number at 100 and varied the Grashof number to measure the effects of the Richardson number. When the Richardson number was 0.001, the flow was found to be strongly influenced by forced convection. At $R i=1$, mixed convection was found to occur. At $R i=100$, the fluid flow was mainly dominated by natural convection. These findings are similar to those of Oztop and Dagtekin [16] even though only one side wall was moving in this case. Chinyoka [20] investigated non-isothermal two-dimensional unsteady fluid flow problems in a rectangular channel.

In this study mixed convection in a two-dimensional cavity is investigated using the penalty-Galerkin finite element method to solve the governing momentum and heat equations. Much work has been done on mixed convection with uniformly heated or cooled walls. Since little work has been done on sinusoidal and linearly heated walls and even less work on a combination of these, the aim of this investigation is to combine linear and sinusoidal heating of different walls of the cavity with a moving lid to study the resulting mixed convective flow. 


\section{Mathematical modeling}

A two-dimensional square cavity of length $L$ is considered for the current investigation. The cavity lid is sinusoidally heated while moving at a constant speed from left to right, and the cavity is heated at a constant rate from the bottom. Two cases of side wall heating are investigated. In the first instance, the cavity side walls are linearly heated, while in case 2 the side walls are uniformly cooled.

The equations that describe the fluid flow and the heat transfer characteristics within the cavity, subject to the Boussinesq approximation are as follows:

$$
\begin{aligned}
& \frac{\partial u}{\partial x}+\frac{\partial v}{\partial y}=0, \\
& u \frac{\partial u}{\partial x}+v \frac{\partial u}{\partial y}=-\frac{1}{\rho} \frac{\partial p}{\partial x}+v\left(\frac{\partial^{2} u}{\partial x^{2}}+\frac{\partial^{2} u}{\partial y^{2}}\right), \\
& u \frac{\partial v}{\partial x}+v \frac{\partial v}{\partial y}=-\frac{1}{\rho} \frac{\partial p}{\partial y}+v\left(\frac{\partial^{2} v}{\partial x^{2}}+\frac{\partial^{2} v}{\partial y^{2}}\right)+g \beta\left(T_{h}-T_{c}\right), \\
& u \frac{\partial T}{\partial x}+v \frac{\partial T}{\partial y}=\alpha\left(\frac{\partial^{2} T}{\partial x^{2}}+\frac{\partial^{2} T}{\partial y^{2}}\right),
\end{aligned}
$$

subject to the boundary conditions

$$
\begin{aligned}
& u=v=0, \quad T=T_{h}-\left(T_{h}-T_{c}\right) \frac{y}{L} \quad \text { or } \quad T=T_{c} \quad \text { at } x=0,1, \\
& u=v=0, \quad T=T_{h} \quad \text { at } y=0, \\
& u=U_{0}, \quad v=0, \quad T=\left(T_{h}-T c\right) \sin \left(\pi \frac{x}{L}\right)+T_{c} \quad \text { at } y=1,
\end{aligned}
$$

where $u$ and $v$ are the velocity components in the $x$ and $y$ directions respectively, $\alpha$ is the thermal diffusivity, $\beta$ is the coefficient of thermal expansion, $v$ is the kinematic viscosity, $\rho$ is the mass density, $g$ is the acceleration due to gravity, $T_{h}$ is the highest temperature attained along the bottom wall and the middle of the top wall and $T_{c}$ is the lowest temperature attained at the top corners in case 1 and along the side walls in case 2 .

We non-dimensionalize the equations by using the following change of variables:

$$
X=\frac{x}{L}, \quad Y=\frac{y}{L}, \quad U=\frac{u}{U_{0}}, \quad V=\frac{v}{U_{0}}, \quad \theta=\frac{T-T_{c}}{T_{h}-T_{c}}, \quad P=\frac{p}{\rho U_{0}^{2}},
$$

where $U_{0}$ is the velocity of the upper wall and $\theta$ is the non-dimensional fluid temperature. This leads to the equations

$$
\begin{aligned}
& \frac{\partial U}{\partial X}+\frac{\partial V}{\partial Y}=0, \\
& U \frac{\partial U}{\partial X}+V \frac{\partial U}{\partial Y}=-\frac{\partial P}{\partial X}+\frac{1}{R e}\left(\frac{\partial^{2} U}{\partial X^{2}}+\frac{\partial^{2} U}{\partial Y^{2}}\right), \\
& U \frac{\partial V}{\partial X}+V \frac{\partial V}{\partial Y}=-\frac{\partial P}{\partial Y}+\frac{1}{R e}\left(\frac{\partial^{2} V}{\partial X^{2}}+\frac{\partial^{2} V}{\partial Y^{2}}\right)+\frac{G r}{R e^{2}} \theta, \\
& U \frac{\partial \theta}{\partial X}+V \frac{\partial \theta}{\partial Y}=\frac{1}{\operatorname{RePr}}\left(\frac{\partial^{2} \theta}{\partial X^{2}}+\frac{\partial^{2} \theta}{\partial Y^{2}}\right),
\end{aligned}
$$




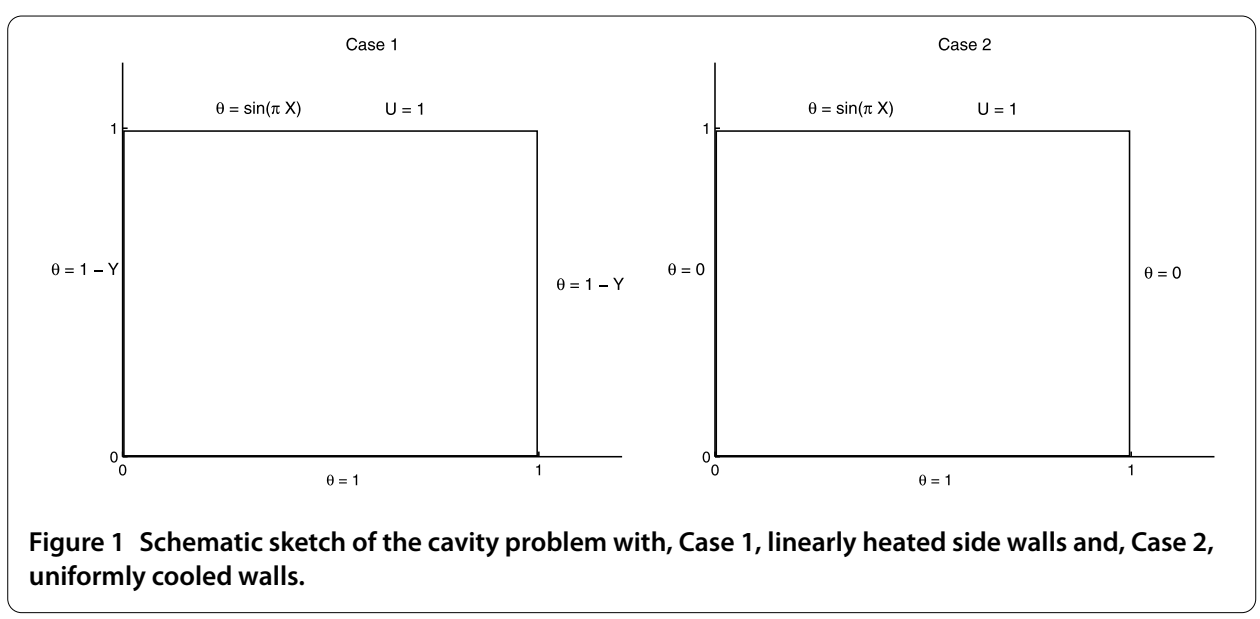

where the important parameters are the Prandtl number $P r$, the Reynolds number $R e$, and the Grashof number $\mathrm{Gr}$ given, respectively, by

$$
\operatorname{Pr}=\frac{v}{\alpha}, \quad \operatorname{Re}=\frac{U_{0} L}{v} \quad \text { and } \quad G r=\frac{g \beta\left(T_{h}-T_{c}\right) L^{3}}{v^{2}} .
$$

The corresponding boundary conditions are:

$$
\begin{aligned}
& U=V=0, \quad \theta=1-Y \quad \text { or } \quad \theta=0 \quad \text { at } X=0,1, \\
& U=V=0, \quad \theta=1 \quad \text { at } Y=0, \\
& U=1, \quad V=0, \quad \theta=\sin (\pi X) \quad \text { at } Y=1 .
\end{aligned}
$$

The geometry of the problem together with the associated boundary conditions is shown in Figure 1.

\section{Numerical solution}

Equations (8)-(11) were solved using the penalty-Galerkin finite element method (PGFEM). This method has the advantage of eliminating pressure as a dependent variable while still satisfying the continuity equation.

We introduce test functions $\phi_{i}$ (which are piecewise, once differentiable functions) and multiply equations (8)-(11) by the test functions to obtain the weak forms of the equations, namely

$$
\begin{aligned}
& \int \phi_{i}\left[U \frac{\partial U}{\partial X}+V \frac{\partial U}{\partial Y}+\frac{\partial P}{\partial X}-\frac{1}{R e}\left(\frac{\partial^{2} U}{\partial X^{2}}+\frac{\partial^{2} U}{\partial Y^{2}}\right)\right] \partial \Omega=0, \\
& \int \phi_{i}\left[U \frac{\partial V}{\partial X}+V \frac{\partial V}{\partial Y}+\frac{\partial P}{\partial Y}-\frac{1}{R e}\left(\frac{\partial^{2} V}{\partial X^{2}}+\frac{\partial^{2} V}{\partial Y^{2}}\right)+\frac{G r}{R e^{2}} \theta\right] \partial \Omega=0, \\
& \int \phi_{i}\left[U \frac{\partial \theta}{\partial X}+V \frac{\partial \theta}{\partial Y}-\frac{1}{\operatorname{RePr}}\left(\frac{\partial^{2} \theta}{\partial X^{2}}+\frac{\partial^{2} \theta}{\partial Y^{2}}\right)\right] \partial \Omega=0 .
\end{aligned}
$$

The continuity equation in its weighted form becomes

$$
\int_{\Omega} \phi_{i}\left(\frac{\partial U}{\partial X}+\frac{\partial V}{\partial Y}\right) d \Omega=0
$$


The PGFEM supposes that we may replace equation (18) with

$$
\int_{\Omega} \phi_{i}\left(\frac{\partial U}{\partial X}+\frac{\partial V}{\partial Y}\right) d \Omega=-\epsilon \int \phi_{i} P
$$

where $\epsilon$ is an arbitrarily small parameter. Rearranging these equations, we find

$$
P=-\lambda \nabla \cdot \mathbf{u},
$$

where $\lambda$ is the penalty parameter. Mass conservation is satisfied in the limit $\varepsilon \rightarrow 0$ or $\lambda \rightarrow \infty$. Here $\lambda$ is chosen to be $10^{7}$ as this has been shown to give consistent results (Basak et al. [14]). If required, the pressure field can be calculated using equation (20). This generally requires a higher level of convergence than is necessary for the velocity field. In this investigation we have chosen to restrict our results to the stream function and temperature contours. As an alternative to the penalty function technique above, the semi-implicit method for pressure linked equations (SIMPLE) technique may be used; see, for instance, Chinyoka [20-22].

Substituting equation (20) into momentum equations (15) and (16) leads to the penalized momentum equations:

$$
\begin{aligned}
& \int \phi_{i}\left(U \frac{\partial U}{\partial X}+V \frac{\partial U}{\partial Y}\right)+\frac{1}{\operatorname{Re}} \frac{\partial \phi_{i}}{\partial X} \frac{\partial U}{\partial X}+\lambda\left[U \frac{\partial}{\partial X}\left(\frac{\partial \phi_{i}}{\partial X}\right)+V \frac{\partial}{\partial X}\left(\frac{\partial \phi_{i}}{\partial Y}\right)\right] \\
& +\frac{1}{\operatorname{Re}} \frac{\partial \phi_{i}}{\partial Y} \frac{\partial U}{\partial Y}=0, \\
& \int \phi_{i}\left(U \frac{\partial V}{\partial X}+V \frac{\partial V}{\partial Y}\right)-\frac{G r \theta}{\operatorname{Re}^{2}} \phi_{i}+\frac{1}{\operatorname{Re}} \frac{\partial \phi_{i}}{\partial X} \frac{\partial V}{\partial X}+\lambda\left[U \frac{\partial}{\partial Y}\left(\frac{\partial \phi_{i}}{\partial X}\right)+V \frac{\partial}{\partial Y}\left(\frac{\partial \phi_{i}}{\partial Y}\right)\right] \\
& +\frac{1}{\operatorname{Re}} \frac{\partial \phi_{i}}{\partial Y} \frac{\partial V}{\partial Y}=0 .
\end{aligned}
$$

To determine the finite element approximate solution of equations (17), (21) and (22), we discretise the domain into 400 rectangular elements with a total of 441 nodes. In principle, more elements may be used to ensure even greater accuracy of results, but this greatly increases the computation time. Initially, one hundred elements were used, but although the computation of such a formulation was efficient, the accuracy of the solutions was impaired. Four hundred elements were found to give sufficient accuracy without impairing the efficiency of the solution method. There are 361 nodes which are not on a Dirichlet boundary. Associated with each node in the domain is a basis function $\phi_{j}$. For convenience, the same functions $\phi_{i}$ were used as both test and basis functions. These basis functions are chosen to be linear Lagrange elements. We expand $\mathrm{U}, \mathrm{V}$ and $\theta$ as linear combinations of the basis functions over the non-Dirichlet boundary nodes as

$$
U=\sum_{j=1}^{361} U_{j} \phi_{j}, \quad V=\sum_{j=1}^{361} V_{j} \phi_{j}, \quad \theta=\sum_{j=1}^{361} \theta_{j} \phi_{j},
$$

where $U_{j}, V_{j}$ and $\theta_{j}$ are the nodal values of $U, V$ and $\theta$ at the $j$ th node. 
By substituting these expressions for $U, V$ and $\theta$ into equations (17), (21) and (22), we obtain (after manipulation and simplification)

$$
\begin{aligned}
& \sum_{j=1}^{361} U_{j} \int_{\Omega}\left[\left(\sum_{k=1}^{361} U_{k} \phi_{k}\right) \frac{\partial \phi_{j}}{\partial X}+\left(\sum_{k=1}^{361} V_{k} \phi_{k}\right) \frac{\partial \phi_{j}}{\partial Y}\right] \phi_{i} d X d Y \\
& +\lambda\left[\sum_{j=1}^{361} U_{j} \int_{\Omega} \frac{\partial \phi_{i}}{\partial X} \frac{\partial \phi_{j}}{\partial X} d X d Y+\sum_{j=1}^{361} V_{j} \int_{\Omega} \frac{\partial \phi_{i}}{\partial X} \frac{\partial \phi_{j}}{\partial Y} d X d Y\right] \\
& +\frac{1}{R e} \sum_{j=1}^{361} U_{j} \int_{\Omega}\left[\frac{\partial \phi_{i}}{\partial X} \frac{\partial \phi_{j}}{\partial X}+\frac{\partial \phi_{i}}{\partial Y} \frac{\partial \phi_{j}}{\partial Y}\right] d X d Y=0, \\
& \sum_{j=1}^{361} V_{j} \int_{\Omega}\left[\left(\sum_{k=1}^{361} U_{k} \phi_{k}\right) \frac{\partial \phi_{j}}{\partial X}+\left(\sum_{k=1}^{361} V_{k} \phi_{k}\right) \frac{\partial \phi_{j}}{\partial Y}\right] \phi_{i} d X d Y \\
& +\lambda\left[\sum_{j=1}^{361} U_{j} \int_{\Omega} \frac{\partial \phi_{i}}{\partial Y} \frac{\partial \phi_{j}}{\partial X} d X d Y+\sum_{j=1}^{361} V_{j} \int_{\Omega} \frac{\partial \phi_{i}}{\partial Y} \frac{\partial \phi_{j}}{\partial Y} d X d Y\right] \\
& +\frac{1}{R e} \sum_{j=1}^{361} V_{j} \int_{\Omega}\left[\frac{\partial \phi_{i}}{\partial X} \frac{\partial \phi_{j}}{\partial X}+\frac{\partial \phi_{i}}{\partial Y} \frac{\partial \phi_{j}}{\partial Y}\right] d X d Y-\frac{G r}{R e^{2}} \int_{\Omega}\left(\sum_{j=1}^{361} \theta_{j} \phi_{j}\right) \phi_{i} d X d Y=0, \\
& \sum_{j=1}^{361} \theta_{j} \int_{\Omega}\left[\left(\sum_{k=1}^{361} U_{k} \phi_{k}\right) \frac{\partial \phi_{j}}{\partial X}+\left(\sum_{k=1}^{361} V_{k} \phi_{k}\right) \frac{\partial \phi_{j}}{\partial Y}\right] \phi_{i} d X d Y \\
& +\frac{1}{\operatorname{RePr}} \sum_{j=1}^{361} \theta_{j} \int_{\Omega}\left[\frac{\partial \phi_{i}}{\partial X} \frac{\partial \phi_{j}}{\partial X}+\frac{\partial \phi_{i}}{\partial Y} \frac{\partial \phi_{j}}{\partial Y}\right] d X d Y=0 .
\end{aligned}
$$

The set of non-linear equations is solved using the Gaussian quadrature and reduced integration to prevent locking, that is, the deterioration in performance of the numerical scheme as $\lambda \rightarrow \infty$ (see, for instance, Qi et al. [23] and the references therein).

\subsection{The stream function and the Nusselt number}

Results of the fluid motion are usually interpreted in terms of the stream function $\psi$, where

$$
U=\frac{\partial \psi}{\partial Y} \quad \text { and } \quad V=-\frac{\partial \psi}{\partial X}
$$

Expanding the stream function in the same way as we did for $U, V$ and $\theta$, we obtain

$$
\begin{aligned}
& \sum_{j=1}^{361} \psi_{j} \int_{\Omega}\left[\frac{\partial \phi_{i}}{\partial X} \frac{\partial \phi_{j}}{\partial X}+\frac{\partial \phi_{i}}{\partial Y} \frac{\partial \phi_{j}}{\partial Y}\right] d X d Y \\
& +\sum_{j=1}^{361} U_{j} \int_{\Omega} \phi_{i} \frac{\partial \phi_{j}}{\partial Y} d X d Y-\sum_{j=1}^{361} V_{j} \int_{\Omega} \phi_{i} \frac{\partial \phi_{j}}{\partial X} d X d Y=0 .
\end{aligned}
$$

The heat transfer coefficient in terms of the Nusselt number is defined as

$$
N u=-\frac{\partial \theta}{\partial \mathbf{n}}
$$




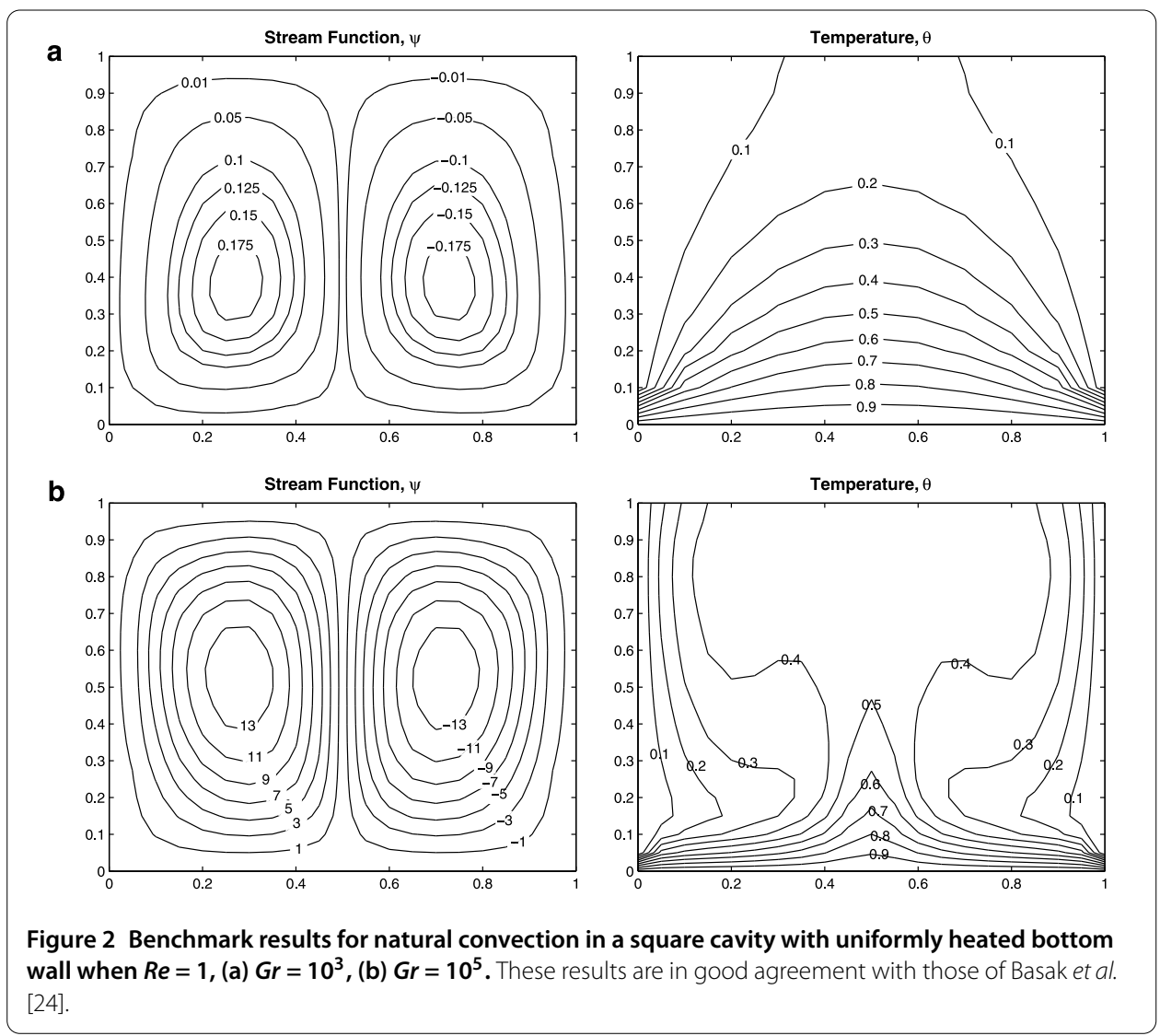

where $\mathbf{n}$ denotes the normal to the plane. The average Nusselt number is defined as

$$
\overline{N u}=\int_{0}^{1} N u d \mathbf{n}
$$

\section{Results and discussion}

Results have been found for $20 \times 20$ quadratic elements with 361 inner nodes, $P r=0.7$ (the value for air), $10^{3} \leq G r \leq 10^{5}$, and $R e=1,10,100$ and 1,000. Convergence was found to be generally poor for $R e>1,000$.

Benchmark results were found for uniformly heated bottom and cooled side walls with an adiabatic stationary lid. Figure 2 shows the stream function and temperature contours for the benchmark results. The results are in good agreement with those in the literature, particularly Basak et al. [24]. We note that in the figures below, a negative stream function value denotes clockwise flow while a positive value denotes anti-clockwise flow.

Figures 3-5 show the results for the stream function and temperature contours for a moving sinusoidally heated lid with linearly heated side walls and a uniformly heated bottom wall for Grashof numbers between $10^{3}$ and $10^{5}$.

Figure 3 shows that for $R e=1, G r=10^{3}$, two counter rotating circulations are formed in the cavity. The circulation on the left-hand side of the cavity is counter-clockwise, while the circulation on the right-hand side of the cavity is clockwise. The clockwise flow is significantly stronger than the anti-clockwise flow and thus it can be observed that the flow is dominated by forced convection due to the moving lid. As was expected, the isotherms for $R e=1, G r=10^{3}$ reach a maximum along the bottom wall and at the center of the top 


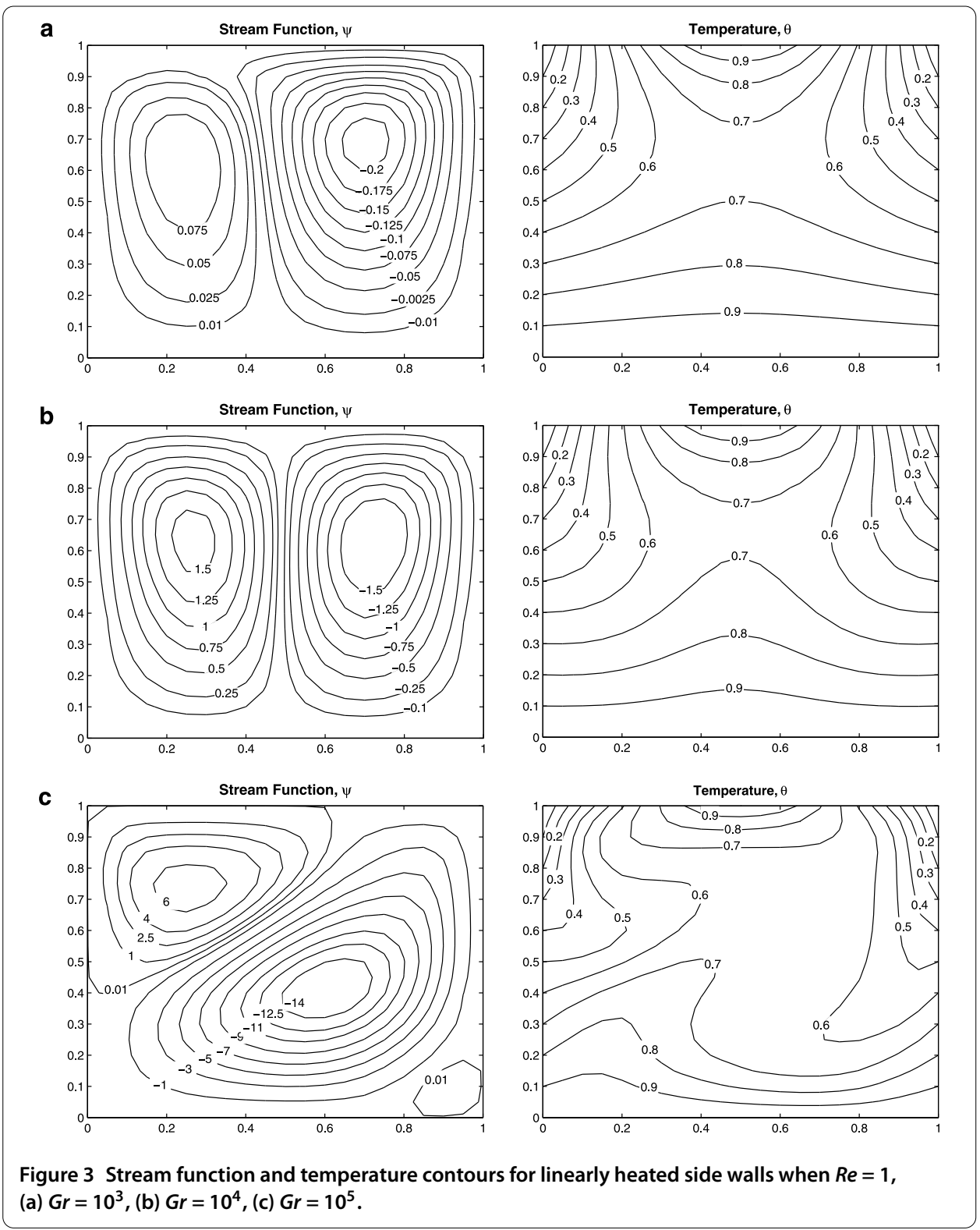

wall, while reaching a minimum at the top corners of the cavity. At the bottom of the cavity, isotherms span the entire width of the cavity. When $\theta \leq 0.6$, the isotherms instead curve upwards towards the lid of the cavity. Hot isotherms at the top of the cavity are restricted to the center of the top wall.

As $G r$ increases to $10^{4}$, the anti-clockwise circulation increases in size and in strength so that two symmetric rotations are formed within the cavity. Natural convection is now as equally dominant as the forced convection regime. The isotherm pattern is similar to that of $G r=10^{3}$, although the heat from the bottom wall has risen slightly higher into the middle of the cavity.

At $G r=10^{5}$, the clockwise circulation in the cavity increases in size and dominates most of the cavity. The circulation is slanted towards the top right and bottom left corners. A small third anti-clockwise circulation is formed on the bottom right-hand side of the cavity. It can be seen that the isotherms are compressed in the top corners of the cavity 


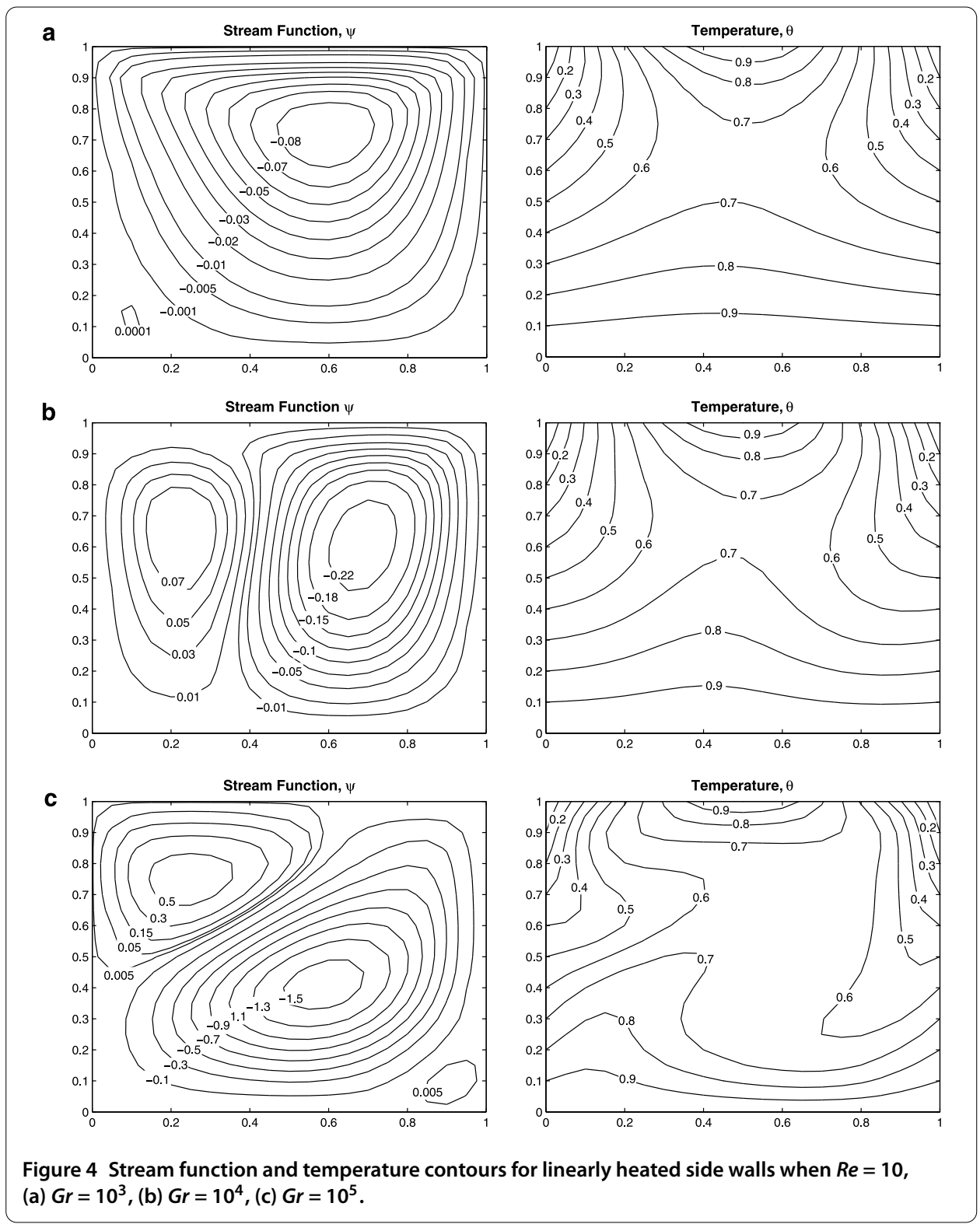

and in the middle of the bottom wall. The isotherms are widely dispersed in the left-hand corner of the cavity. This is due to the shape and angle of the clockwise circulation. Cool fluid is brought down on the right-hand side of the cavity, while hot fluid is transported upwards towards the middle of the cavity on the left-hand side of the bottom wall. The strength of the circulations increase with increasing Grashof numbers.

For $R e=10$ and $G r=10^{3}$ (see Figure 4), the flow in the cavity is clearly dominated by forced convection. Only a very small secondary circulation is found for this low Grashof number. The isotherms are no longer perfectly symmetrical. As Gr increases to $10^{4}$, the secondary circulation on the left-hand side of the cavity grows in size and strength. The strength of the clockwise circulation is stronger than that of the anti-clockwise circulation. The flow is still dominated by lid-driven flow, but natural convection now has a more significant effect on the flow. An increase in the Grashof number causes the heat to rise further towards the center of the cavity. When $G r=10^{5}$, the secondary circulation increases 


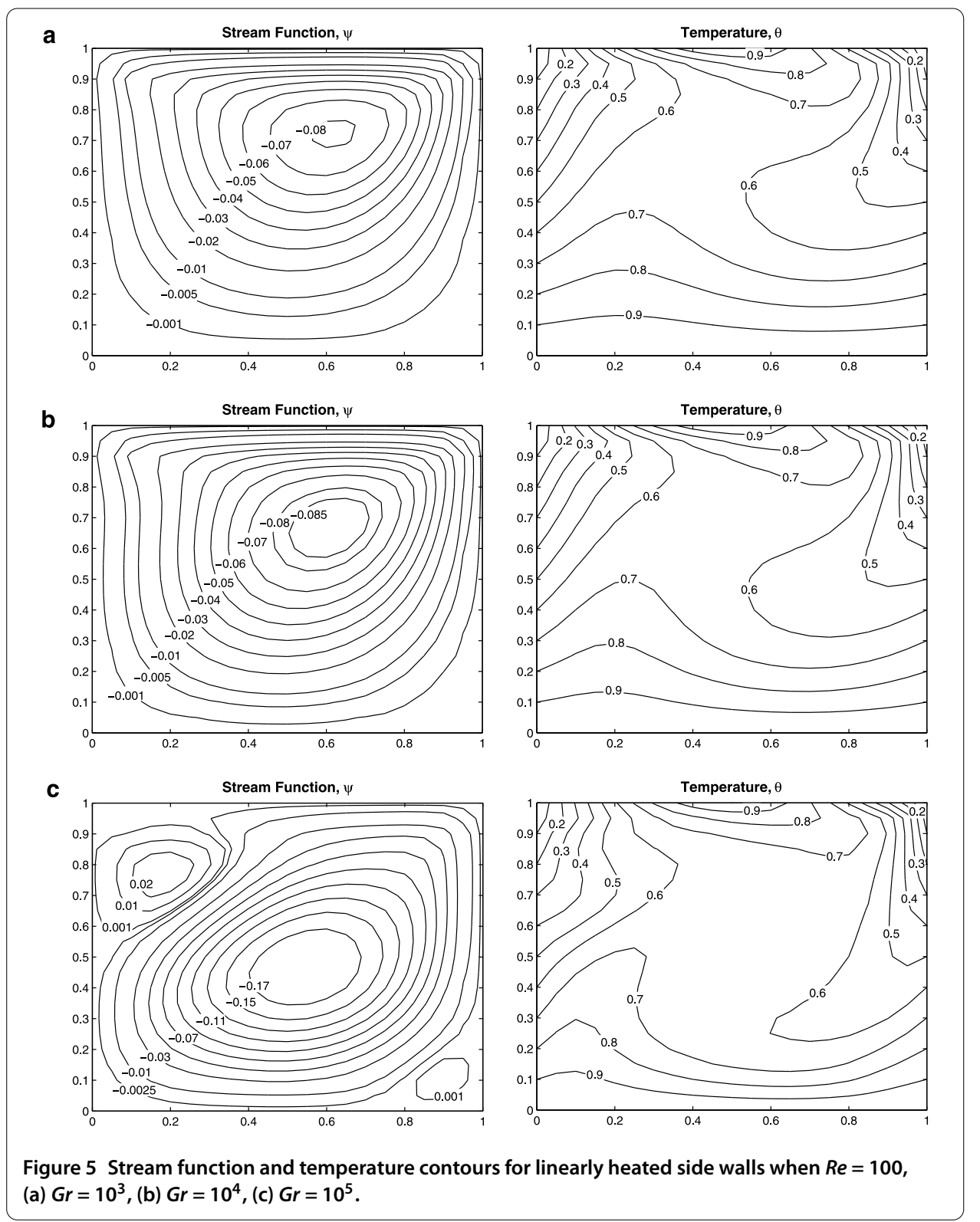

in size, becomes slanted and dominates most of the cavity and a small third clockwise circulation is formed on the bottom right-hand side of the cavity. The isotherms at $G r=10^{5}$ are also similar in shape to those when $R e=1$.

In Figure 5 at $R e=100, G r=10^{3}$ and $G r=10^{4}$, the flow is again dominated by forced convection. A single clockwise circulation is formed due to strong inertial effects at the top wall. There is little change in the flow as the Grashof number increases from $10^{3}$ to $10^{4}$. The isotherms for both $G r=10^{3}$ and $G r=10^{4}$ are dispersed on the left-hand side of the cavity and are compressed on the right-hand side of the cavity. This is due to the domination of the forced convection flow regime. At $R e=100, G r=10^{5}$, a large slanted clockwise circulation is formed which dominates more of the cavity than the circulations when $R e=1$ and when $R e=10$. Again a small third anti-clockwise circulation is formed at the bottom right-hand corner of the cavity. The isotherms are similar in shape for $R e=1$ and $R e=10$. 
a

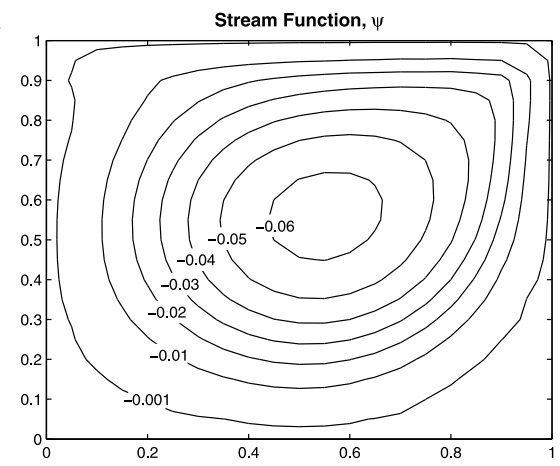

b
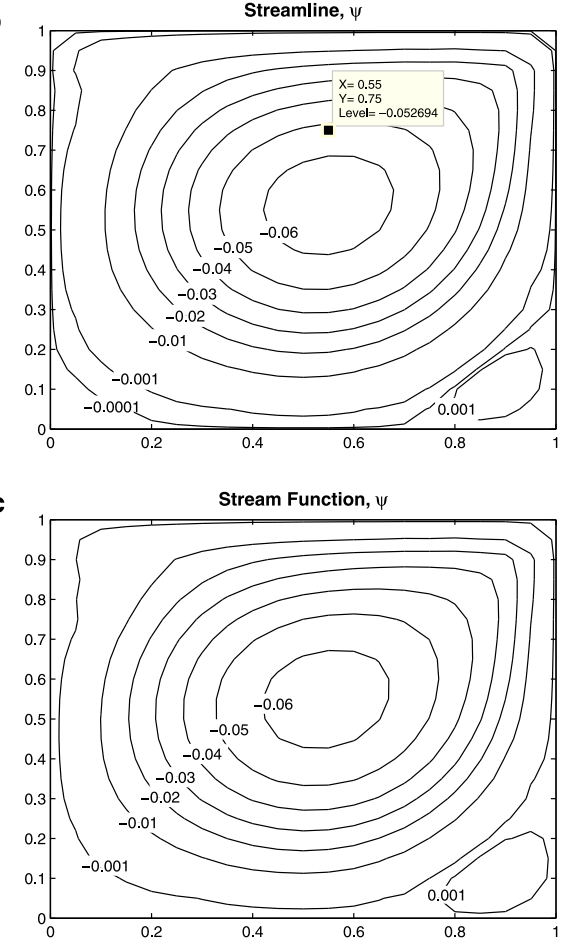
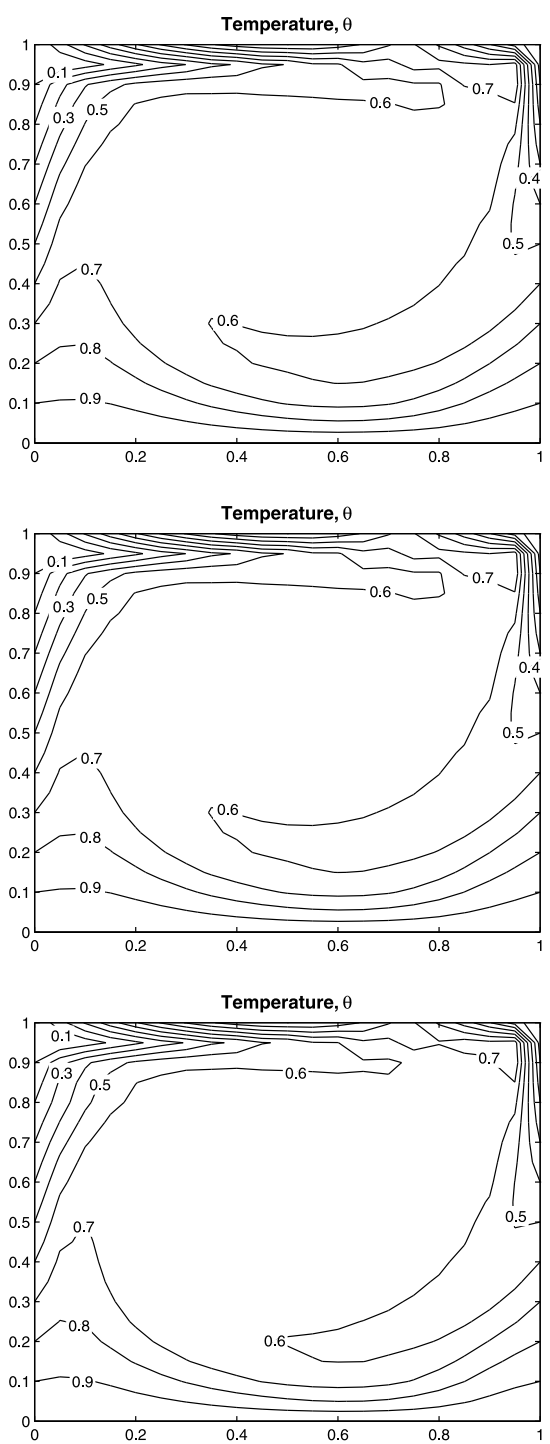

Figure 6 Stream function and temperature contours for linearly heated side walls when $R e=1,000$, (a) $G r=10^{3}$, (b) $G r=10^{4}$, (c) $G r=10^{5}$.

For $R e=1,000$ (see Figure 6), the flow is dominated by forced convection for $G r=10^{3}$, $G r=10^{4}$ and $G r=10^{5}$. For $G r=10^{3}$, a single clockwise circulation is formed within the cavity. As $\mathrm{Gr}$ increases to $10^{4}$, a small anti-clockwise circulation is formed on the bottom right-hand side of the cavity. As Gr increases to $10^{5}$, there is little change in the fluid flow. The isotherms for both $G r=10^{3}$ and $G r=10^{4}$ are dispersed on the bottom lefthand side of the cavity and compressed on the bottom right-hand side of the cavity. The warm isotherms are dispersed towards the top right-hand side of the cavity, causing the cool isotherms to be compressed into the top right corner. As Gr increases to $10^{5}$, the hot isotherms stretch slightly higher into the center of the cavity. It can be seen that as the Reynolds number increases, the strength of the circulation decreases.

Figures 7-9 show the results for the stream function and temperature contours for a moving sinusoidally heated lid with cooled side walls and uniformly heated bottom wall for $\operatorname{Pr}=0.7, R e=1,10$ and 100 and a range of Grashof numbers between $10^{3}$ and $10^{5}$. For 
a
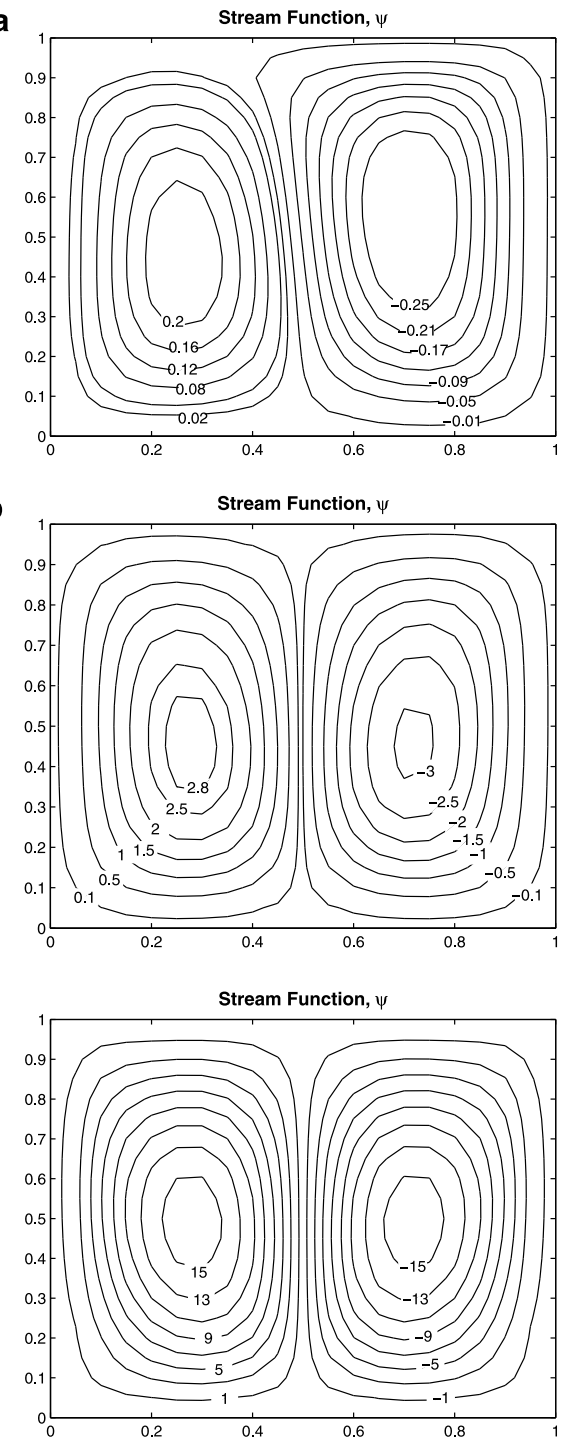

Temperature, $\theta$

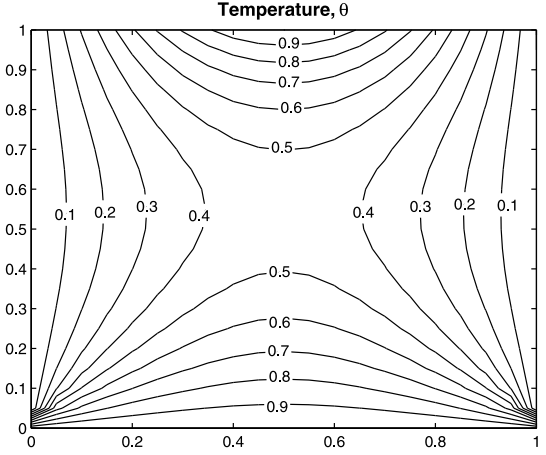

Temperature, $\theta$

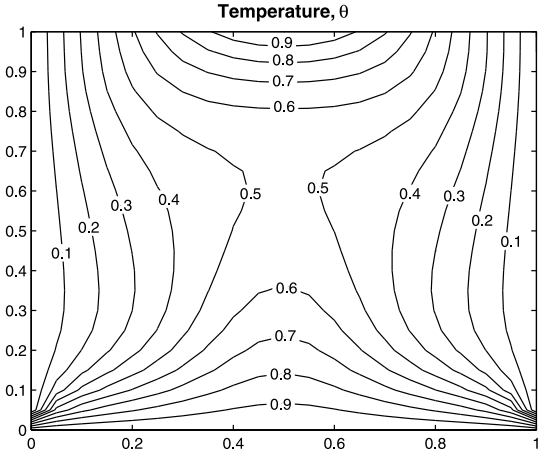

Temperature, $\theta$

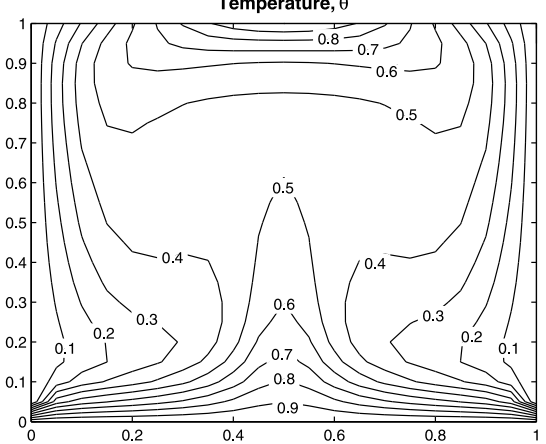

Figure 7 Stream function and temperature contours for cooled side walls when $\operatorname{Re}=1$, (a) $G r=10^{3}$, (b) $G r=10^{4}$, (c) $G r=10^{5}$.

$R e=1$ and $G r=10^{3}$ (Figure 7), the flow is slightly dominated by forced convection. Two counter rotating circulations are formed in the cavity, with the clockwise circulation being only slightly stronger than the anti-clockwise circulation. The temperature distribution across the cavity shows that the cavity is at a high temperature across the bottom of the cavity and towards the center of the top of the cavity. The cavity is cooler towards the side walls and top corners as was expected. The hot isotherms still span the width of the cavity, but are pushed downwards in the corners. For $\theta \geq 0.5$, the isotherms are horizontal across the cavity, but for $\theta \leq 0.4$, the isotherms stretch vertically across the cavity. The temperature distribution obviously differs from that of the linearly heated walls at the side walls. It can also be seen that the contour lines are more widely dispersed towards the bottom of the cavity for the linearly heated case. 


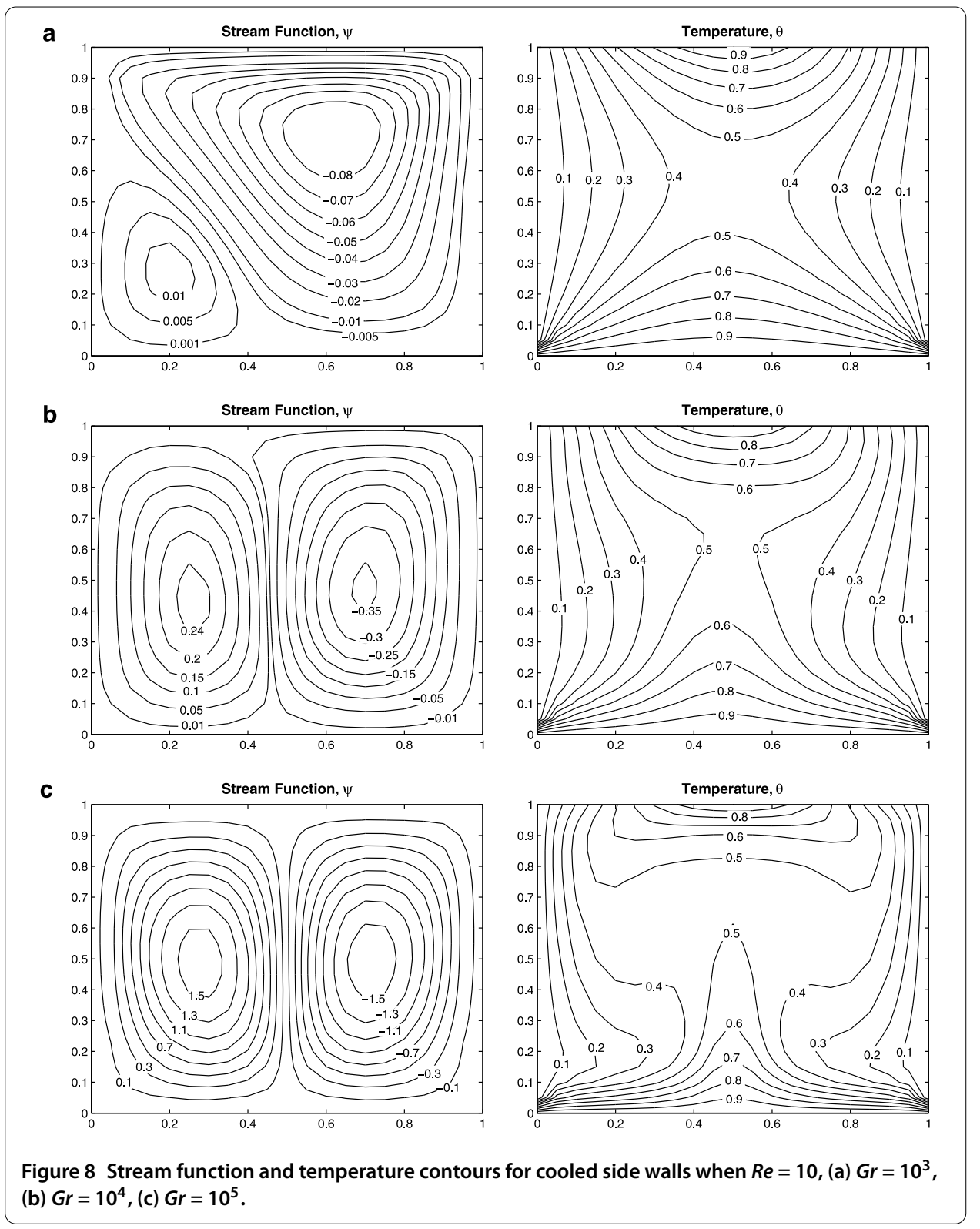

As $G r$ increases to $10^{4}$, the clockwise circulation increases in size to form two counter rotating circulations of a similar size and the natural convection is almost equally dominant to that of forced convection. The centers of the circulations are now at the same height. The temperature contours become slightly more compressed towards the side walls as the Grashof number increases. The hotter contour lines from the top wall are seen to be wider than that of $G r=10^{3}$ and are found to be narrower in the center towards the bottom wall. As $G r$ increases to $10^{5}$, the strength of the stream function increases and the size and position of the two circulations are identical. It can be seen that the hot contour lines have widened even further along the top wall causing the cooler contour lines to become compressed towards the top of the cavity. Towards the bottom of the cavity, the cool isotherms (those where $\theta \leq 0.4$ ) are widely dispersed towards the center of the cavity 


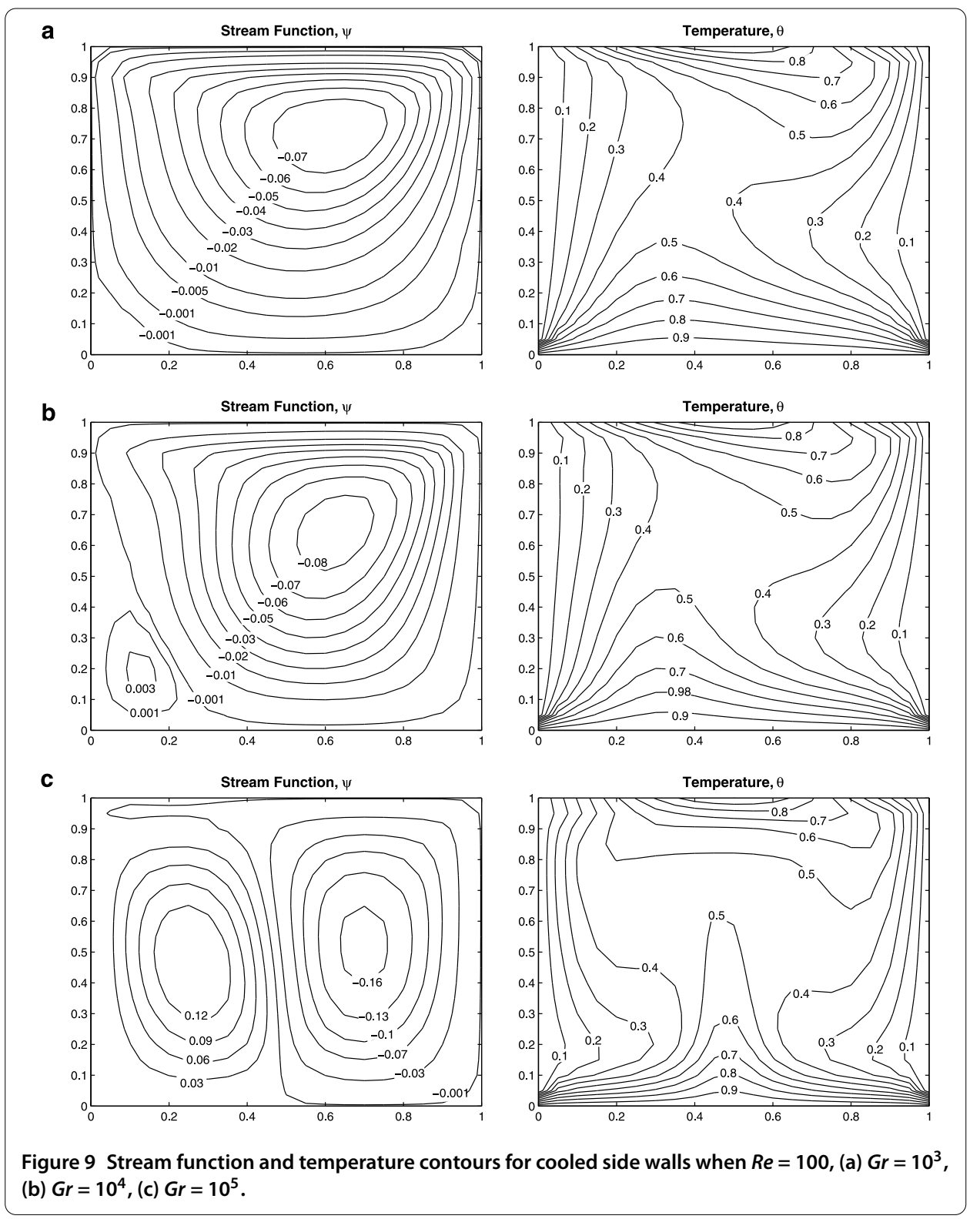

while the warmer isotherms are compressed towards the bottom wall. This is particularly the case towards the bottom corners of the cavity.

In Figure 8 it is clear that for $R e=10, G r=10^{3}$, the flow is dominated by forced convection. The majority of the cavity is dominated by a clockwise rotating circulation. A small anti-clockwise circulation is formed on the bottom left-hand side of the cavity. The temperature contours are similar to the case where $R e=1$. On close inspection it can be seen that the contours are slightly more compressed towards the top right corner than when $R e=1$.

As $G r$ increases to $10^{4}$, the anti-clockwise circulation grows in size although the clockwise rotating circulation is still larger in size and in strength indicating that the flow is still slightly dominated by forced convection. Again the contour lines are similar in shape to those for $R e=1$, and a slight compression towards the top right-hand corner can be seen. As $\mathrm{Gr}$ increases further to $10^{5}$, natural convection becomes equal to forced convection. 
There is no observable difference in the shape of the temperature contours from that of $R e=1$

Figure 9 shows that for $R e=100$ and $G r=10^{3}$ and $G r=10^{4}$, the flow is dominated by forced convection. For $\mathrm{Gr}=10^{3}$, only one clockwise rotating circulation appears in the cavity. As $G r$ increases to $10^{4}$, a small anti-clockwise circulation is formed in the bottom left-hand corner of the cavity. As $G r$ increases to $10^{5}$, the anti-clockwise circulation increases in size and strength. The flow is still slightly dominated by forced convection. The temperature contours for $R e=100$ show a marked difference to those for $R e=1$ and $R e=10$. For $G r=10^{3}$, the hot isotherms (where $\theta \geq 0.5$ ) are dispersed towards the bottom left and top right corners and compressed in the top left and bottom right corners. The cool isotherms are compressed in the top right and bottom left corners and dispersed towards the center of the cavity along the middle and bottom of the right wall and at the top of the left wall. The compression and dispersion is more significant along the right side wall than along the left side wall. As $G r$ increases to $10^{4}$, the hot temperature contours from the bottom wall move slightly higher towards the center of the cavity, while the basic shape of the contours remains the same as those for $G r=10^{3}$. A further increase in $G r$ to $10^{5}$ causes the temperature contours to become compressed in the top two corners of the cavity and more dispersed in the bottom two corners. There is a significant compression of isotherms towards the top right-hand corner. The hot temperature contours from the bottom wall become more elongated and extend further towards the center of the cavity.

Figure 10 shows that for $R e=1,000, G r=10^{3}$ and $G r=10^{4}$, the flow is dominated by forced convection with a single clockwise circulation being formed within the cavity. As Gr increases to $10^{5}$, an anti-clockwise circulation is formed on the bottom left-hand side of the cavity. The isotherms for $\mathrm{Gr}=10^{3}$ and $\mathrm{Gr}=10^{4}$ are similar. The hot isotherms are dispersed on the left-hand side of the cavity and compressed on the right-hand side of the cavity. Warm isotherms ( $\theta=0.5$ and 0.6$)$ from the top of the cavity are stretched downwards towards the right-hand side of the cavity. As Gr increases to $10^{5}$, the warm isotherms stretch slightly higher into the center of the cavity than for $G r=10^{3}$ and $G r=10^{4}$. The warm isotherms from the top of the cavity stretch slightly further downwards towards the center right-hand side of the cavity.

In general, the circulation is stronger for the case of cooled side walls than that for linearly heated walls. The anti-clockwise circulation is significantly stronger for the cooled walls than for the linearly heated walls while the clockwise circulation is only slightly stronger for the cooled walls. For $R e=1$ and 10 , the cooled walls produce two identical counter rotating circulations in contrast to the three tilted circulations resulting from the linearly heated walls. For $G r=10^{5}$, the stream function pattern for the cooled walls shows a slightly stronger clockwise circulation indicating a slight domination by forced convection, but again the flow pattern is different from that of the linearly heated side walls.

\section{Heat transfer at the walls}

Figure 11 shows the effect of the Grashof number on the heat transfer at each of the walls in the cavity for the case of linearly heated side walls.

For all values of the Grashof number, the local Nusselt number is equal to one at the edge of the bottom wall on both sides due to the linear heating of the side walls. For $G r=10^{3}$, the local Nusselt number falls below 1 and reaches a local minimum between $x=0.4$ and $x=0.5$. There is not much variation in the Nusselt number across the cavity due to the 


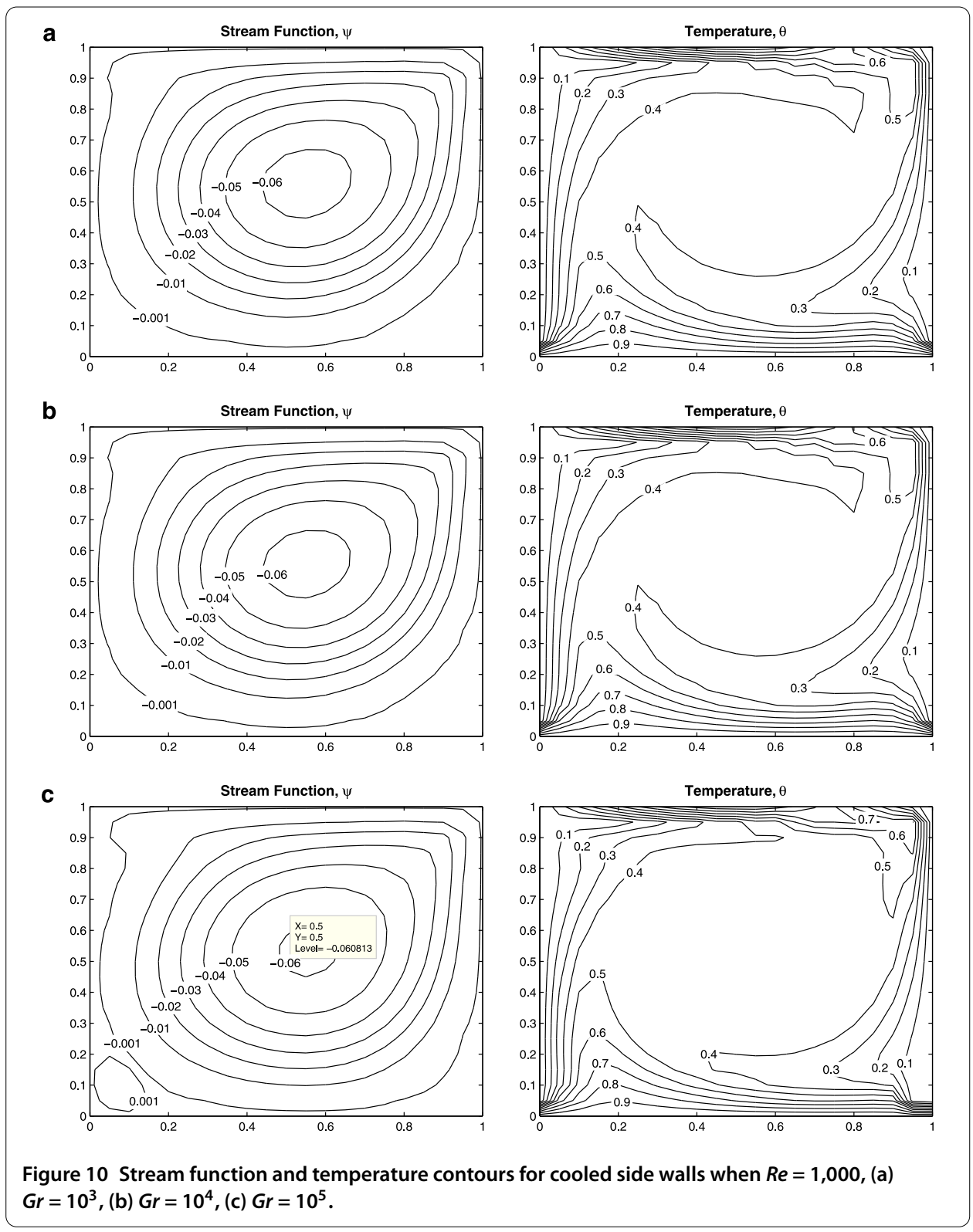

relatively flat isotherms at the bottom of the cavity. When $G r=10^{4}$, the Nusselt number is of a similar value to that for $G r=10^{3}$ between $x=0$ and $x=0.5$. Beyond $x=0.6$, the graph rises above that of $G r=10^{3}$ reaching a maximum at $x=0.8$.

When $G r=10^{5}$, the Nusselt number is greater than 1 for the majority of the cavity due to the compressed isotherms. The Nusselt number drops below 1 between $x=0$ and $x=0.2$ due to the widely dispersed isotherms on the left-hand side of the cavity. The Nusselt number is at a maximum at $x=0.7$. The local Nusselt number at the edges of the top wall is equal to -1 for all values of the Grashof number due to the linearly heated walls. For $G r=10^{3}$, the Nusselt number rises and reaches a local maximum at $x=0.4$ and then gradually decreases. For $G r=10^{4}$, the Nusselt number follows a similar pattern to that for $G r=10^{3}$, although is lower than the graph of $G r=10^{3}$. At $G r=10^{5}$, the graph of the Nusselt number is close to being symmetric in shape. The Nusselt number drops to a local 


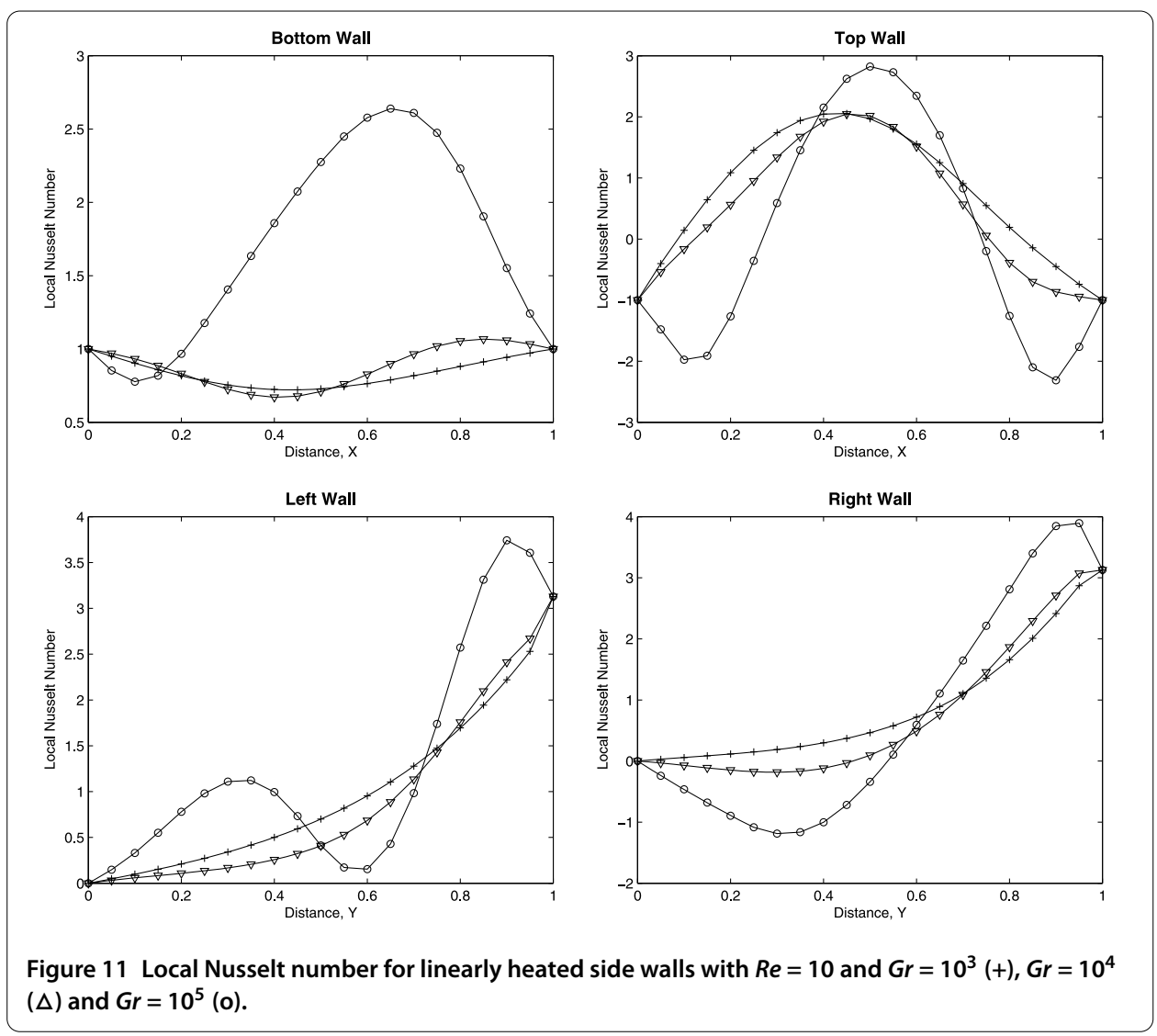

minimum at $x=0.1$ and then rises to a local maximum at the center of the cavity. The Nusselt number then drops again to a local minimum at $x=0.9$. When $G r=10^{3}$, the local Nusselt number at the right-hand wall gradually increases as $y$ increases. For $G r=10^{4}$, a similar occurrence happens, although the Nusselt number is below that for $G r=10^{3}$ until $y=0.8$ where the Nusselt number becomes greater than that of $G r=10^{3}$. For $G r=10^{5}$, the Nusselt number decreases below 0 attaining a local minimum at $y=0.3$ due to the compressed isotherms at the bottom corner of the right wall. The Nusselt number then increases reaching a local maximum at $y=0.9$.

The local Nusselt number at the left-hand wall for $G r=10^{3}$ and $G r=10^{4}$ is very similar in shape to that for the right-hand wall due to a near symmetrical isotherm pattern at the two walls. However at $G r=10^{5}$, the local Nusselt number at the left-hand wall is above 0 for the entire height of the cavity and has an oscillatory pattern. The Nusselt number initially rises attaining a local maximum at $y=0.3$ due to the dispersed isotherms along the bottom half of the left-hand wall. Towards the top of the cavity, the isotherms become more dispersed once again and the Nusselt number again rises to a local maximum at $y=0.9$.

The graph at the left-hand wall is similar to that of Basak et al. [15] which also exhibits a slightly oscillatory shape. However, the graph in the present study has a local maximum near the top corner and then decreases in contrast to the graph in Basak et al. [15] which increases monotonically to the wall. This is due to the difference between the adiabatic and the heated lid. 


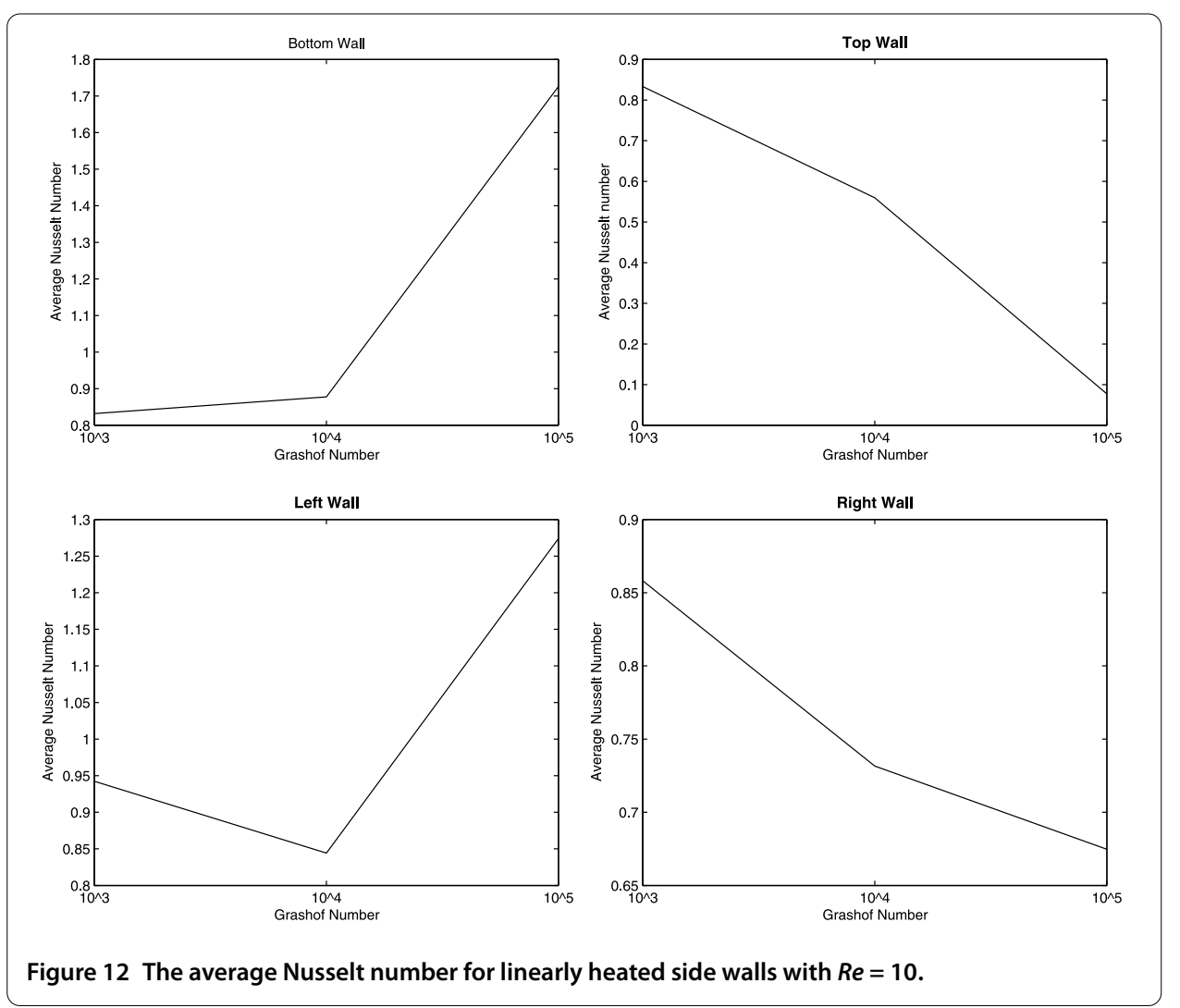

Figure 12 shows the average Nusselt number at the four walls of the cavity. At the bottom wall, the Nusselt number is seen to increase as the Grashof number increases. The graph of the average Nusselt number at the bottom wall is almost identical to the results of Basak et al. [15]. At the top wall, the opposite is seen to happen. This is due to the fact that as the Grashof number increases, the isotherms become more compressed in the top corners. At the left-hand side wall, the average Nusselt number is lower for $\mathrm{Gr}=10^{4}$ than it is for $G r=10^{3}$. This is due to the slight dip in the isotherms at the left wall. The average Nusselt number at the left wall then increases for $G r=10^{5}$. The average Nusselt number at the right side wall decreases as the Grashof number increases. The local Nusselt number at the bottom wall for cooled side walls is shown in Figure 13. For all values of the Grashof number, the Nusselt number decreases to $x=0.5$ where it attains a local minimum. The local Nusselt number when $G r=10^{3}$ is lower than that when $G r=10^{4}$ which, in turn, is lower than that when $G r=10^{5}$. In contrast to the case of linearly heated side walls, the local Nusselt number at the bottom wall for cooled walls is concave up due to the fact that the isotherms are equal to 0 at the corners of the bottom wall for the cooled walls.

The local Nusselt number decreases to a local minimum at $y=0.6$ for $G r=10^{3}$ and then increases slightly towards the top of the cavity. For $G r=10^{4}$, the Nusselt number decreases to a local minimum near $y=0.3$ and then slowly increases towards the top of the cavity. When $G r=10^{5}$, the local Nusselt number decreases to a local minimum near $y=0.2$ due to the dispersion of isotherms near the bottom corner(s). The Nusselt number then increases above that of $G r=10^{4}$ and $G r=10^{3}$ at $y=0.25$ and $y=0.3$, respectively, due to the more compressed isotherms in the top corners. The Nusselt number then increases to a local 


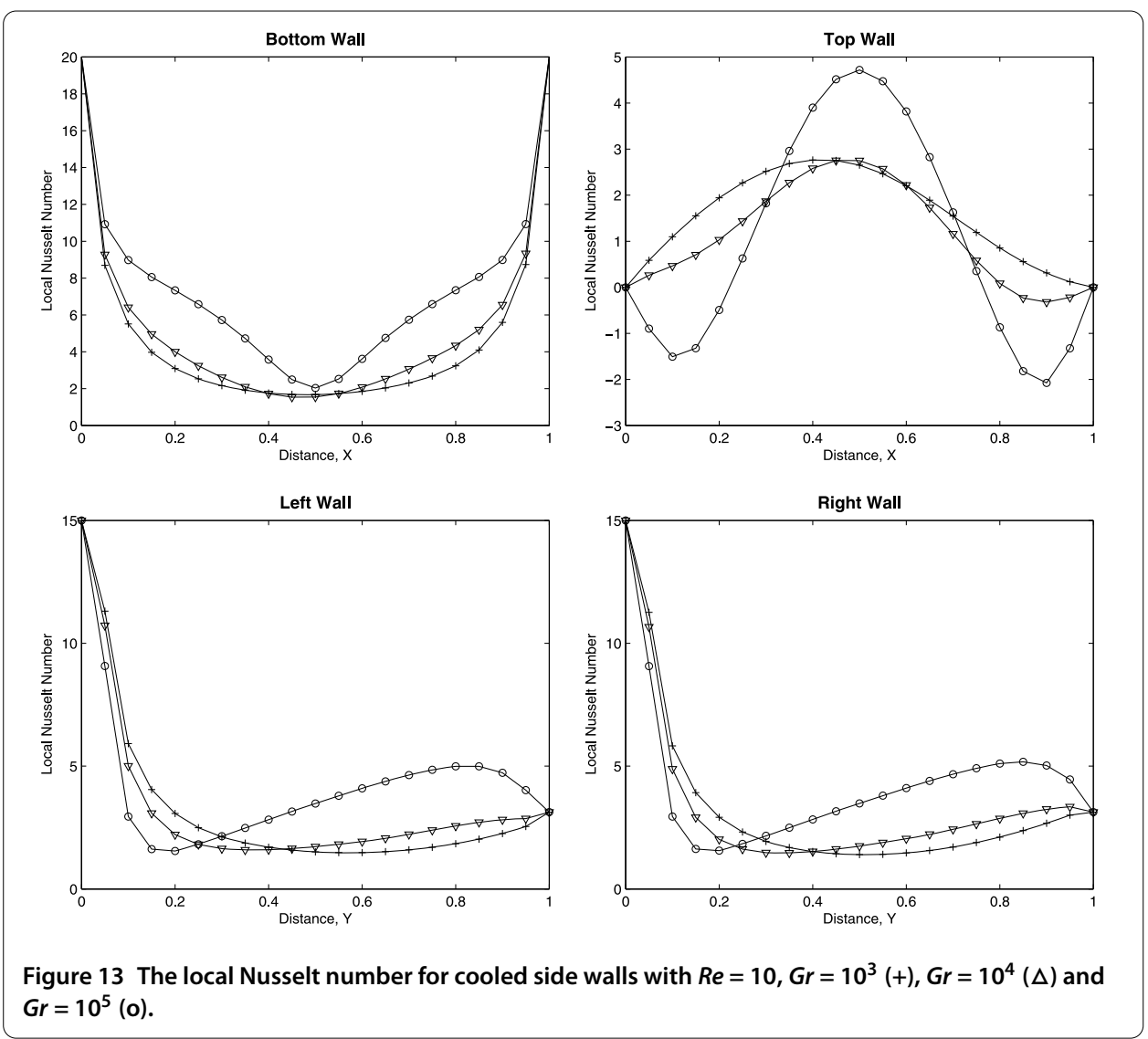

maximum near $y=0.8$. There is no oscillatory pattern when $G r=10^{5}$ as was observed in the case of the heated side walls.

\section{Conclusion}

A numerical study has been performed using the penalty-Galerkin finite element method to analyze mixed convective heat transfer and fluid flow in an air filled square cavity. The effects of linearly heated and uniformly cooled walls on flow and heat transfer characteristics within the cavity have been studied. It was observed that

- In general, the strength of circulation was stronger for the case of cooled walls, and that the anti-clockwise circulation was significantly stronger for cooled walls, while the clockwise circulation was only slightly stronger for cooled walls. The difference in strength decreased with increasing both Reynolds and Grashof numbers.

- Both the local and average Nusselt numbers were generally higher for the case of cooled side walls than those for heated side walls.

- The local Nusselt numbers at the left and right walls were similar in the case of cooled side walls due to symmetric patterns in the temperature isotherms. However, particularly for $G r=10^{5}$, the heated side walls had different local Nusselt numbers at the two different walls.

- The local Nusselt number at the top wall was similar in shape for the two cases although the linearly heated case was lower in value than for the cooled side walls. 


\section{Competing interests}

The authors declare that they have no competing interests.

\section{Authors' contributions}

DSD carried out the numerical computations. PS participated in the design of the study and helped to draft the manuscript.

\section{Acknowledgements}

The authors are grateful to the University of KwaZulu-Natal for financial support.

\section{Received: 18 September 2012 Accepted: 18 March 2013 Published: 10 April 2013}

\section{References}

1. Torrance, K, Davis, R, Eike, K, Gill, P, Gutman, D, Hsui, A, Lyons, S, Zien, H: Cavity flows driven by buoyancy and shear. J. Fluid Mech. 51, 221-231 (1972)

2. Kawaguti, M: Numerical solution of the Navier-Stokes equations for the flow in a two-dimensional cavity. J. Phys. Soc. Jpn. 16, 2307-2315 (1961)

3. Burggraf, OR: Analytical and numerical studies of the structure of steady separated flows. J. Fluid Mech. 24, 113-151 (1966)

4. Heinrich, JC, Marshall, RS: Viscous incompressible flow by a penalty function finite element method. Comput. Fluids 9, 73-83 (1981)

5. Eckert, EG, Carlson, WO: Natural convection in an air layer enclosed between two vertical plates with different temperatures. Int. J. Heat Mass Transf. 2, 106-110 (1961)

6. Ostrach, S: Natural convection in enclosures. Adv. Heat Transf. 7, 161-227 (1972)

7. Prasad, AK, Koseff, JR: Combined forced and natural convection heat transfer in a deep lid-driven cavity flow. Int. J. Heat Fluid Flow 17, 460-467 (1996)

8. Moallemi, M, Jang, KS: PrandtI number effects on laminar mixed convection heat transfer in a lid driven cavity. Int. J. Heat Mass Transf. 35, 1881-1892 (1992)

9. Sivakumar, V, Sivasankaran, S, Prakash, P, Lee, J: Effect of heating location and size on mixed convection in lid-driven cavities. Comput. Math. Appl. 59, 3053-3065 (2010)

10. Nithyadevi, N, Kandaswamy, P, Lee, J: Natural convection in a rectangular cavity with partially active side walls. Int. J. Heat Mass Transf. 50, 4688-4697 (2007)

11. Paraconi, M, Corvaro, F: Natural convection in a square enclosure with a hot source. Int. J. Therm. Sci. 48, 1683-1695 (2009)

12. Pesso, T, Piva, S: Laminar natural convection in a square cavity: low Prandtl numbers and large density differences. Int. J. Heat Mass Transf. 52, 1036-1043 (2009)

13. Sathiyamoorthy, M, Basak, T, Roy, S, Mahanti, NC: Effect of the temperature difference aspect ratio on natural convection in a square cavity for nonuniform thermal boundary conditions. J. Heat Transf. 129, 1723-1728 (2007)

14. Basak, T, Roy, S, Sharma, PK, Pop, I: Analysis of mixed convection flows within a square cavity with uniform and non-uniform heating of bottom wall. Int. J. Therm. Sci. 48, 891-912 (2009)

15. Basak, T, Roy, S, Sharma, PK, Pop, I: Analysis of mixed convection flows within a square cavity with linearly heated side wall(s). Int. J. Heat Mass Transf. 52, 2224-2242 (2009)

16. Oztop, H, Dagtekin, I: Mixed convection in two-sided lid-driven differentially heated square cavity. Int. J. Heat Mass Transf. 47, 1761-1769 (2004)

17. Cheng, TS, Liu, WH: Effect of temperature gradient orientation on the characteristics of mixed convection flow in a lid-driven square cavity. Comput. Fluids 39, 965-978 (2010)

18. Corcione, M: Effects of the thermal boundary conditions at the sidewalls upon natural convection in rectangular enclosures heated from below and cooled from above. Int. J. Therm. Sci. 42, 199-208 (2003)

19. Wong, JC: Numerical simulation of two-dimensional laminar mixed-convection in a lid-driven cavity using the mixed finite element consistent splitting scheme. Int. J. Numer. Methods Heat Fluid Flow 17, 46-93 (2007)

20. Chinyoka, T: Two-dimensional flow of chemically reactive viscoelastic fluids with or without the influence of thermal convection. Commun. Nonlinear Sci. Numer. Simul. 16, 1387-1395 (2011)

21. Chinyoka, T: Viscoelastic effects in double-pipe single-pass counter flow heat exchangers. Int. J. Numer. Methods Fluids 59, 677-690 (2009)

22. Chinyoka, T: Modelling of cross-flow heat exchangers with viscoelastic fluids. Nonlinear Anal., Real World Appl. 10 3353-3359 (2009)

23. Qi, H, Wang, L-H, Zheng, W-Y: On locking-free finite element schemes for three-dimensional elasticity. J. Comput. Math. 23, 101-112 (2005)

24. Basak, T, Roy, S, Balakrishnan, AR: Effects of thermal boundary conditions on natural convection flows within a square cavity. Int. J. Heat Mass Transf. 49, 4525-4535 (2006) 